\title{
Effects of a short interval carbohydrate free diet on tissue FDG uptake
}

\author{
Daniel Klein ${ }^{1}$, J ason DiPoce ${ }^{1}$, Dmitry Volkin ${ }^{2}$, Arnold Brenner ${ }^{1,3}$ \\ 1. Department of Radiology, Staten Island University Hospital, Staten Island, NY, USA. 2. SUNY Downstate Medical
} School, Brooklyn, NY, USA. 3. Department of Nuclear Medicine, Albert Einstein College of Medicine, Bronx, NY, USA

Correspondence: Daniel Klein. Address: 475 Seaview Avenue Staten Island, NY 10305, USA. Telephone: 01-718-226-8297. Email: Dklein143@gmail.com.

Received: November 27, 2012

Accepted: May 28, $2013 \quad$ Online Published: July 17, 2013

DOI : $10.5430 /$ jbgc.v3n4p54

URL: http://dx.doi.org/10.5430/jbgc.v3n4p54

\section{Abstract}

Purpose: Tight glucose control is essential for 18-Fludeoxyglucose (18F-FDG) positron emission tomography (PET) imaging. A strategy to ensure appropriate glucose levels is to advise a carbohydrate free diet prior to scanning. This alters glucose metabolism which can have ramifications on tissue FDG biodistribution. The aim of this study is to evaluate the effects of advising a carbohydrate free diet for two days prior to PET scan on FDG uptake in the liver, heart, bone marrow, skeletal muscle and tumor.

Materials and methods: All patients at an outpatient facility were advised to maintain a diet free of carbohydrates for two days prior to 18F- FDG PET/Computed tomography (CT) scanning to maintain glucose control at the time of the scan. Self reported adherence to the diet was recorded by the scheduling coordinator on the day of imaging. A retrospective analysis of 228 consecutive 18F-FDG PET/CT scans was performed in which the maximum standardized uptake value (max SUV) was recorded for the following six sites: right and left hepatic lobes, left ventricle of the heart, sacrum, thigh and tumor. The max SUV of the patients who adhered to the diet $(n=144)$ were compared with those who did not $(n=85)$.

Results: The average max SUV for the right and left hepatic lobes and the left ventricle were $3.93 \pm 0.78,3.70 \pm 0.76$ and $6.34 \pm 5.12$ for the adherent patients and $3.63 \pm 0.84,3.41 \pm 0.80$ and $6.98 \pm 4.69$ for the non-adherent patients. The average max SUV in the hepatic lobes of the adherent group was statistically significantly higher by $8.2 \%$ in the right lobe and $8.8 \%$ in left lobe when compared with the non-adherent group $(p<0.003)$. The average max SUV in the heart of the adherent group was statistically significantly lower by $10 \%$ when compared with the non-adherent group $(p<0.040)$. No significant difference was observed in the remaining tissues, including tumor.

Conclusions: Altering carbohydrate intake can significantly affect the metabolic milieu (FDG biodistribution in normal tissues). The liver max SUV in patients on a carbohydrate free diet prior to PET scanning was higher than those who were not. This difference should be kept in mind when defining a normal cut off threshold SUV in the liver.

\section{Key words}

Positron emission tomography, Standardized uptake value, High Protein low carbohydrate diet metabolism

\section{I ntroduction}

18F-FDG is a positron labeled glucose analogue. 18F-FDG enters the cell under the influence of cell membrane glucose receptors. FDG-6-Phosphate accumulates in the cell since it cannot be further metabolized. Cancer cells demonstrate an 
increase in glucose transport proteins such as GLUT-1 and increased levels or activity of hexokinase that leads to increased uptake and retention of $18 \mathrm{~F}-\mathrm{FDG}^{[1,2]}$. Additionally there is increased glycolysis in cells with increased metabolic demand ${ }^{[3]}$. This characteristic is utilized during 18F-FDG imaging to evaluate biologic tumor activity.

Normal tissue will take up 18F-FDG, albeit to a lesser extent than tumor. It is sometimes difficult to differentiate normal from abnormal tissue ${ }^{[4]}$. For this reason optimal glucose metabolism is needed during PET imaging. Patients with diabetes mellitus pose a challenge given frequent elevated serum glucose levels on the day of imaging. Multiple publications have demonstrated detrimental effects of a high serum glucose level on tumor FDG uptake ${ }^{[5,6]}$. Moreover, multiple studies document a lower standardized uptake value (SUV) in tumors found in patients with elevated serum glucose levels than those who do not ${ }^{[7,8]}$. This is mainly caused by direct competition between 18 F-FDG and glucose for tumor uptake ${ }^{[9]}$. To prevent this potential influence on imaging, serum glucose levels are routinely obtained from patients at the time of 18F-FDG imaging and those with elevated levels may need to be rescheduled. This may pose a scheduling conflict and financial implications since the radiopharmaceutical is wasted. Multiple strategies have been employed to ensure optimal glucose control on the day of imaging. At our institution we ask all patients to maintain a low carbohydrate diet for 1-2 days prior to the exam.

The metabolic milieu is altered when patients are on a high protein and low carbohydrate diet (HPLC). There is decreased fasting and postprandial serum glucose levels, increased serum triglycerides, decreased serum insulin levels and increased gluconeogenesis within the liver ${ }^{[10,11]}$. Multiple studies document altered 18F-FDG biodistribution after a HPLC diet. Brown fat and cardiac uptake decrease when patients are on such a diet, and utilizing a HPLC diet may decrease uptake in these normal structures and help to optimize image interpretation ${ }^{[12]}$. Our experience shows that patients on a HPLC diet 1-2 days prior to 18F-FDG PET scanning have better glucose control on the day of imaging. It is conceivable that this altered metabolism will affect tissue 18F-FDG biodistribution. The aim of our study is to compare normal and tumor 18F-FDG biodistribution between patients who have maintained a HPLC diet 1-2 days prior to scanning and those who have continued their regular diet. Cutoff values are commonly utilized to distinguish normal from pathological radiotracer uptake. The effects of a HPLC diet on tissue 18F-FDG biodistribution may alter tissue specific max SUV for detection of biological tumor activity in organs that are normally visualized on 18F-FDG imaging.

\section{Material and methods}

All patients scheduled to receive an 18F-FDG PET/CT scan at our outpatient facility are informed to maintain a diet high in protein and low in carbohydrates for two days prior to their scheduled scan. Patients are advised by the scheduling coordinator as to which foods to avoid and which foods to consume based on standard low carbohydrate diet guidelines. On the day of the exam the coordinator records which foods the patients consumed over the prior two days and whether they adhered to the advised diet. A retrospective review of 228 consecutive patients (age $64.6 \pm 12.4$ ) receiving 18F-FDG PET/CT scans at our institution was performed. This study was approved by our institutional review board.

\subsection{PET procedure}

FDG PET images were acquired with a whole-body Discovery ST PET/CT scanner (General Electric Medical Systems, Milwaukee, WI) about 50 minutes after the administration of 18F-FDG of 296-444 MBq (8-12 mCi). All patients fasted for at least 6 hours prior to the study. Patients were maintained in a quiet room for approximately 50 minutes prior to scanning. Patients were positioned in the supine position on the scanner bed, and emission scans centered on the $511 \mathrm{keV}$ photon of 18 Fluoride were obtained as 5 minute in duration bed position acquisitions (5-6 bed positions per patient) from skull base to proximal thigh. A low dose CT scan was obtained immediately prior to emission imaging for attenuation correction and image fusion. Tomographic reconstructions were generated for both coregistered CT and emission images. Images were reviewed on an Xeleris 1.0 workstation (GE). 


\subsection{Data acquisition}

In addition to the tumor (when available) non-pathological regions were chosen to represent normal tissue biodistribution. These sites included; the right and left lobes of the liver, left ventricle, sacrum, and thigh (see Figure 1). Maximum standardized uptake value (maxSUV) was utilized to quantify tissue 18F-FDG uptake. Regions of interest were chosen over each anatomic site (coronal images) and maxSUV was recorded. Data was recorded using Microsoft Excel.

\subsection{Statistical analysis}

Results are expressed as mean \pm standard deviation for numerical values and as "yes or no" for diet adherence. Comparison between the maxSUV of the specified tissue groups for the patients who adhered to the diet were compared with those who did not using the $t$ and Mann-Whitney tests. A probability value ( $p$-value) less than 0.05 was considered statistically significant.

Figure 1. Non-pathological regions chosen to represent normal tissue biodistribution (white circles). These sites included; the right and left lobes of the liver, left ventricle, sacrum (bone marrow), and thigh (skeletal
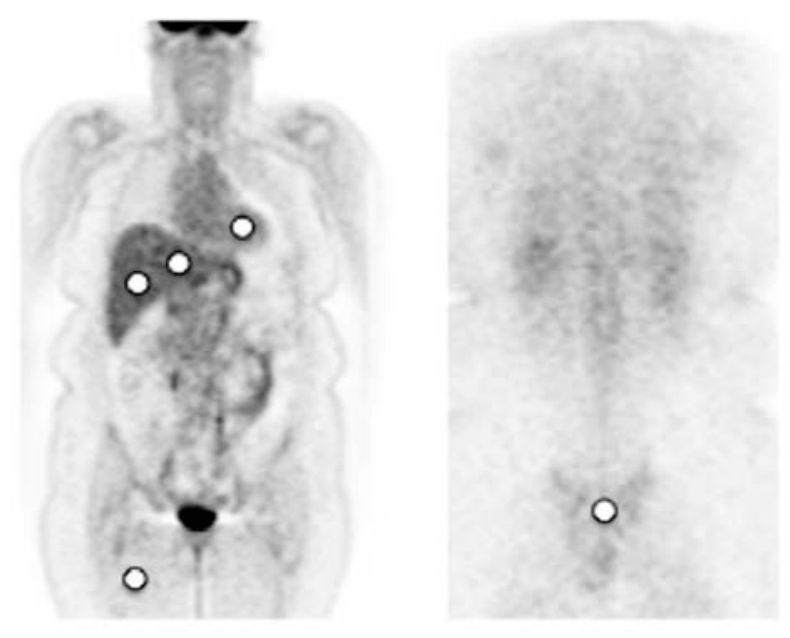
muscle).

\section{Results}

\subsection{Patient characteristics}

Of the 228 patients studied, 144 (62.9\%) adhered to the diet and 85 (37.1\%) did not. Of the 144 patients who adhered to the diet, 101 specified the number of days that they were on the diet - 39 for 1 day and 62 for 2 days. The overall mean serum glucose level was $120.73 \mathrm{mg} / \mathrm{dL}$ (67-201). Table 1 shows the mean age and serum glucose levels for each group.

Table 1. Patient characteristics

\begin{tabular}{llll}
\hline & Diet Adherent Group & Diet Non-Adherent Group & $\boldsymbol{p}$-value \\
\hline Age (years) & $64.79 \pm 11.46$ & $64.29 \pm 13.84$ & 0.896 \\
Serum Glucose (mg/dL) & $120.37 \pm 27.01$ & $121.36 \pm 29.88$ & 0.979 \\
\hline
\end{tabular}

\subsection{Tissue average maxSUV between the two groups}

Table 2 shows the average maxSUV for the respective tissues between the two groups. There was a statistically significant increase in average maxSUV in patients on the HPLC diet with regard to the right ( $8.2 \%$ increase, $p<0.003)$ and left lobes of the liver ( $8.8 \%$ increase, $p<0.003)$. There was a statistically significant decrease in average maxSUV in patients on the 
HPLC diet with regard to the left ventricle (10\% decrease, $p<0.040)$. No significant difference was identified within the other tissues including tumor.

Table 2. Average maxSUV of respective tissues

\begin{tabular}{llll}
\hline Tissue & Diet Adherent Group Average maxSUV & Diet Non-Adherent Group Average maxSUV & $\boldsymbol{p}$-value \\
\hline Right Lobe of Liver & $3.93 \pm 0.78$ & $3.63 \pm 0.84$ & 0.001 \\
Left Lobe of Liver & $3.70 \pm 0.76$ & $3.41 \pm 0.80$ & 0.002 \\
Left Ventricle & $6.34 \pm 5.12$ & $6.98 \pm 4.69$ & 0.034 \\
Sacrum & $2.07 \pm 0.51$ & $2.06 \pm 0.61$ & 0.749 \\
Right Thigh & $1.03 \pm 0.26$ & $0.98 \pm 0.25$ & 0.515 \\
\hline
\end{tabular}

\section{Discussion}

This study highlights the relationship of glucose metabolism and tissue 18F-FDG uptake in the liver and left ventricle. We demonstrated that a short interval HPLC diet significantly increased maxSUV in the liver and decreased maxSUV in the left ventricle.

Multiple studies have shown a decrease in left ventricular maxSUV on HPLC diets ${ }^{[13]}$. Harisanker et al. reported a decrease in left ventricular maxSUV from 5.06 to $3.09^{[14]}$. Our study reinforces the fact that a HPLC diet can decrease left ventricular maxSUV. A proposed explanation for this decrease is that when patients are on a HPLC diet there is increased access to fatty acids and hence reduced glucose metabolism. Some studies have shown greater myocardial suppression when fatty loading is added to the low carbohydrate diet by having the patients increase fat consumption ${ }^{[15,16]}$.

To our knowledge, no study has demonstrated an effect on normal liver FDG uptake with HPLC diets. The reason for the increased uptake in the liver is unclear. Documented changes in the liver during a short interval low carbohydrate diet include depletion of glycogen stores, increased gluconeogenesis and production of ketone bodies ${ }^{[17,18]}$.

Our results indicate that a patient's diet can affect normal tissue uptake even if the diet was only for a short period of time. In everyday practice interpreting physicians must differentiate normal from pathological tissue uptake in organs that are normally visualized on 18F-FDG imaging. Sometimes this is based on a cut off SUV value ${ }^{[19]}$. A simple dietary change can affect this threshold as we have demonstrated in the liver.

Although not a primary objective in our study, we did not demonstrate a statistically significant difference in tumor uptake between the groups. This fact is reassuring given that maxSUV in tumor has been shown to be a prognostic indicator ${ }^{[20-22]}$.

Our study has multiple limitations. Our patients were not on strictly controlled diets. Although strict instructions were given to the patients, the actual food consumption was not monitored aside from self-reporting. Additionally, some patients indicated that they were on the low carbohydrate diet for two days prior to the study while others only one day. Other patient characteristics that may affect glucose metabolism such as documented diabetes or serum triglyceride levels were not available for our patient population and may have affected the results.

\section{Conclusions}

Altering carbohydrate intake can significantly affect the metabolic milieu (FDG biodistribution in normal tissues). The liver max SUV in patients on the carbohydrate free diet prior to PET scanning was significantly higher than those who were not. This difference should be kept in mind when defining a normal threshold maxSUV in the liver. 


\section{References}

[1] Som P, Atkins HL, Bandoypadhyay D, et al. A fluorinated glucose analog, 2-fluoro-2-deoxy-D-glucose (F-18): nontoxic tracer for rapid tumor detection. J Nucl Med. 1980; 21: 670-675. PMid:7391842

[2] Alakus H, Batur M, Schmidt M, et al. Variable 18F-fluorodeoxyglucose uptake in gastric cancer is associated with different levels of GLUT-1 expression. Nucl Med Commun. 2010; 31: 532-538. PMid:20220543

[3] Warburg O. On the origin of cancer cells. Science. 1956; 123: 309-314. PMid:13298683 http://dx.doi.org/10.1126/science.123.3191.309

[4] Shreve PD, Anzai Y, Wahl RL. Pitfalls in oncologic diagnosis with FDG PET imaging: physiologic and benign variants. Radiographics. 1999; 19: 61-77; quiz 150-151. PMid:9925392

[5] Wahl RL, Henry CA, Ethier SP. Serum glucose: effects on tumor and normal tissue accumulation of 2-[F-18]-fluoro-2-deoxy-Dglucose in rodents with mammary carcinoma. Radiology. 1992; 183: 643-647. PMid:1584912

[6] Rabkin Z, Israel O, Keidar Z. Do hyperglycemia and diabetes affect the incidence of false-negative 18F-FDG PET/CT studies in patients evaluated for infection or inflammation and cancer? A Comparative analysis. J Nucl Med. 2010; 51: 1015-1020. PMid:20554733 ttp://dx.doi.org/10.2967/jnumed.109.074294

[7] Langen KJ, Braun U, Rota Kops E, et al. The influence of plasma glucose levels on fluorine-18-fluorodeoxyglucose uptake in bronchial carcinomas. J Nucl Med. 1993; 34: 355-359. PMid:8441023

[8] Travaini L, Trifiro G, Paganelli G. [18F] FDG uptake: pay attention to candies. Ecancermedicalscience. 2007; 1: 48. PMid:22275952

[9] Torizuka T, Zasadny KR, Wahl RL. Diabetes Decreases FDG Accumulation in Primary Lung Cancer. Clin Positron Imaging. 1999; 2: 281-287. http://dx.doi.org/10.1016/S1095-0397(99)00029-1

[10] Adam-Perrot A, Clifton P, Brouns F. Low-carbohydrate diets: nutritional and physiological aspects. Obes Rev. 2006 ; 7: 49-58. PMid:16436102 http://dx.doi.org/10.1111/j.1467-789X.2006.00222.x

[11] Bravata DM, Sanders L, Huang J, Krumholz HM, Olkin I, Gardner CD. Efficacy and safety of low-carbohydrate diets: a systematic review. JAMA. 2003; 289: 1837-1850. PMid:12684364 http://dx.doi.org/10.1001/jama.289.14.1837

[12] Williams G, Kolodny GM. Method for decreasing uptake of 18F-FDG by hypermetabolic brown adipose tissue on PET. AJR Am J Roentgenol. 2008; 190: 1406-1409. PMid:18430862 http://dx.doi.org/10.2214/AJR.07.3205

[13] Balink H, Hut E, Pol T, Flokstra FJ, Roef M. Suppression of 18F-FDG Myocardial Uptake Using a Fat-Allowed, CarbohydrateRestricted Diet. J Nucl Med Technol. 2011; 39: 185-189. PMid:21795368 http://dx.doi.org/10.2967/jnmt.110.076489

[14] Harisankar CN, Mittal BR, Agrawal KL, Abrar ML, Bhattacharya A. Utility of high fat and low carbohydrate diet in suppressing myocardial FDG uptake. J Nucl Cardiol. 2011; 18: 926-936. PMid:21732228 http://dx.doi.org/10.1007/s12350-011-9422-8

[15] Williams G, Kolodny GM. Suppression of myocardial 18F-FDG uptake by preparing patients with a high-fat, low-carbohydrate diet. AJR Am J Roentgenol. 2008; 190: W151-156. PMid:18212199 http://dx.doi.org/10.2214/AJR.07.2409

[16] Fox JJ, Strauss HW. One step closer to imaging vulnerable plaque in the coronary arteries. J Nucl Med. 2009 ; 50: 497-500. PMid:19289437 http://dx.doi.org/10.2967/jnumed.108.056325

[17] Bilsborough SA, Crowe TC. Low-carbohydrate diets: what are the potential short- and long-term health implications? Asia Pac J Clin Nutr. 2003; 12: 396-404. PMid:14672862

[18] Boden G, Sargrad K, Homko C, Mozzoli M, Stein TP. Effect of a low-carbohydrate diet on appetite, blood glucose levels, and insulin resistance in obese patients with type 2 diabetes. Ann Intern Med. 2005; 142: 403-411. PMid:15767618 http://dx.doi.org/10.7326/0003-4819-142-6-200503150-00006

[19] Ramos CD, Erdi YE, Gonen M, et al. FDG-PET standardized uptake values in normal anatomical structures using iterative reconstruction segmented attenuation correction and filtered back-projection. Eur J Nucl Med. 2001; 28: 155-164. PMid:11303885 http://dx.doi.org/10.1007/s002590000421

[20] Spaepen K, Stroobants S, Dupont P, et al. Prognostic value of positron emission tomography (PET) with fluorine-18 fluorodeoxyglucose ([18F]FDG) after first-line chemotherapy in non-Hodgkin's lymphoma: is [18F]FDG-PET a valid alternative to conventional diagnostic methods? J Clin Oncol. 2001; 19: 414-419. PMid:11208833

[21] Vansteenkiste JF, Stroobants SG, Dupont PJ, et al. Prognostic importance of the standardized uptake value on (18)F-fluoro-2-deoxy-glucose-positron emission tomography scan in non-small-cell lung cancer: An analysis of 125 cases. Leuven Lung Cancer Group. J Clin Oncol. 1999; 17: 3201-3206. PMid:10506619

[22] Benard F, Sterman D, Smith RJ, Kaiser LR, Albelda SM, Alavi A. Prognostic value of FDG PET imaging in malignant pleural mesothelioma. J Nucl Med. 1999; 40: 1241-1245. PMid:10450672 